\title{
Érotisme et création ou la mort en sursis
}

John Fletcher

\section{(2) OpenEdition}

\section{Journals}

Édition électronique

URL : https://journals.openedition.org/ccs/1005

DOI : $10.4000 /$ ccs. 1005

ISSN : 2558-782X

\section{Éditeur :}

Presses universitaires de Rennes, Association des lecteurs de Claude Simon

\section{Édition imprimée}

Date de publication : 1 décembre 2017

Pagination : $69-78$

ISBN : $978-2-7535-5482-5$

ISSN : 1774-9425

\section{Référence électronique}

John Fletcher, «Érotisme et création ou la mort en sursis », Cahiers Claude Simon [En ligne], 12 | 2017, mis en ligne le 07 décembre 2018, consulté le 25 avril 2022. URL : http://journals.openedition.org/ccs/ 1005 ; DOI : https://doi.org/10.4000/ccs.1005 


\title{
ÉROTISME ET CRÉATION OU LA MORT EN SURSIS ${ }^{1}$
}

\author{
John FLETCHER \\ University of Kent
}

La vie humaine ne peut suivre sans trembler - sans tricher - le mouvement qui l'entraîne à la mort.

Georges Bataille

Dans son ouvrage le plus récent, Orion aveugle, Claude Simon accorde une place importante à une eau-forte de Picasso exécutée en 1968 et qui reprend un sujet favori, obsessionnel même du peintre, celui de l'artiste et son modèle. Ceux-ci se trouvent enlacés dans une étreinte lascive et provocante, d'une grande beauté formelle; et c'est à la lumière de cette œuvre, si proche des préoccupations du romancier, que j'aimerais tenter de dégager l'un des thèmes les plus insistants de la création simonienne.

En effet, il est impossible de ne pas être de plus en plus sensible à la force du thème érotique chez Claude Simon, et ceci amène le lecteur à se poser une question. S'agit-il de la recherche d'une totalité, d'une manière d'envisager l'expérience humaine qui permettrait d'y discerner une harmonie profonde entre les divergentes impulsions de l'être, comme ce fut le cas, par exemple, chez Lou Andreas-Salomé? D’après Dominique Fernandez, en effet, cette passionnée de psychanalyse freudienne «trouva enfin le moyen de concilier l'esprit et le sexe, de s'expliquer à partir de quelle origine commune jaillissent l'inspiration artistique et l'exaltation amoureuse » (Nouvel

1. Écrit entre la parution d'Orion aveugle et celle des Corps conducteurs, l'article a été publié dans la revue Entretiens, no 31 ("Claude Simon»), 1972, p. 131-140. Seule modification avec le texte original: le recours pour les citations aux éditions de référence en vigueur dans les Cahiers $(N d E)$. 
Observateur, 11 janvier 1971). Et Georges Bataille pouvait affirmer à son tour, "j'ai tout sacrifié à la recherche d'un point de vue d'où ressorte l'unité de l'esprit humain " (L'Érotisme, U.G.E., «10/18», 1964, p. 10).

Est-on en train d'assister au même mouvement chez Claude Simon? S'achemine-t-il vers cette totalité vers laquelle tendraient Lou Andreas-Salomé et Georges Bataille, et si bien exprimée par Picasso dans l'eau-forte tant appréciée par le romancier - œuvre qui représente le mythe éternel du peintre, palette à la main, sur le point de posséder une femme belle, pâmée et amoureuse dont le portrait en cours d'achèvement repose sur le chevalet, son exécution momentanément interrompue par un élan où l'adoration esthétique cède le pas à cette jouissance érotique qui en est le fondement? Est-ce ainsi que le roman simonien voit le rapport entre érotisme et création? C'est bien le cas chez d'autres écrivains, dont Apollinaire et Durrel, pour ne citer qu'eux. Ou s'agitil au contraire, de tout autre chose? D'une disjonction, où érotisme et création seraient en fin de compte inconciliables, radicalement opposés? Où le jeu romanesque tendrait à disloquer les termes de la belle image de la genèse que nous devons à Rimbaud: " la mer allée avec le soleil "? Voilà les questions que je me suis posées, auxquelles je m'efforcerai de répondre au cours de cet essai. Mais peut-être devrions-nous d'abord nous pencher sur quelques faits précis tels que nous les découvrons au fil de l'œuvre simonienne; une fois les données clairement établies, nous serons sans doute en mesure de les interpréter à la lumière des principes déjà évoqués.

Le rôle joué par la femme dans les romans de Claude Simon, s'il est parfois équivoque, n'en reste pas moins essentiel. Ce ne peut être un hasard si le romancier a publié, avec le concours d'un peintre à qui il voue une grande admiration, Joan Miró, un ouvrage intitulé Femmes; comme les illustrations de Miró, les femmes simoniennes sont toujours énigmatiques, presque indéchiffrables. On peut néanmoins en esquisser une typologie sommaire. Il s'agit, d'une part, de la jeune fille raide, gauche et innocente: on se souviendra de Belle, du Tricheur, et de Véra, la jolie Russe de La Corde raide, appartenant à la même catégorie, qui est décrite en ces termes:

Mais elle ne savait pas embrasser, pas plus qu'elle ne savait faire l'amour, raide et se cachant la figure dans ses bras, quand elle se donna, d'une façon brusque, tard dans la 
nuit. Il n'y avait pas de draps à son lit, et pour éteindre, il fallait dévisser l'ampoule de la douille. (CR, p. 16)

Les métamorphoses de Véra, avec sa beauté irritante, ses manières brusques et la façon touchante dont elle pleure en prenant congé du narrateur, sont multiples. Nous la retrouvons dans Gulliver sous les traits d'Éliane, et dans Le Sacre du Printemps sous ceux d'Édith, obligée d'avoir recours au vol pour se procurer un avortement. Elle renaît sous les traits de Cécile du Vent, qui tombe amoureuse de Montès, et à défaut de celui-ci se donne sans aucune passion au jeune imbécile à qui sa famille la destine. C'est elle encore qui, " déflorée parmi les fleurs sylvestres ", entreprend " maladroitement " d'essuyer le membre ensanglanté du narrateur d'Histoire (p. 375); elle, toujours, qui gêne tellement le narrateur de La Bataille de Pharsale avec ses "gestes brusques d'adolescent poussé trop vite» (p. 646); et elle, enfin, "qui n'a jamais tenu un homme embrassé, [dont le ventre] n’a jamais enfanté ", la vieille tante Marie de L'Herbe (p. 141). Vierges folles ou vierges douloureusement déflorées, ces jeunes et vieilles filles sont autant d'avatars d'une seule et même figure qui hante les pages de cette œuvre.

L'autre visage est celui de la femme voluptueuse, pleine d'expérience, dévorante et quelque peu putain, voire nymphomane, comme la Pasiphaé de la mythologie grecque dont le taureau-amant parle crûment dans La Bataille de Pharsale:

comment avec de si grosses et si longues pattes pourrais-je chevaucher un corps si délicat comment avec mes durs sabots étreindre des membres si blancs si tendres faits de lait et de miel et les petites lèvres empourprées par une rosée d'ambroisie comment les baiser d'une bouche aussi large que la mienne monstrueuse avec ses dents difformes rocheuses et puis enfin comment une femme même en feu jusqu'au bout des ongles pourrait-elle jamais accueillir un membre aussi énorme et elle pendant ce temps multipliait les mots tendres ses furieux baisers ses doux gémissements ses yeux me mordaient Je te tiens me dit-elle dans un paroxysme je tiens mon petit pigeon mon passereau et alors combien mes imaginations avaient été fausses et mes craintes stupides elle me le prouva car m'embrassant plus étroitement encore c'est tout entier oui tout entier qu'elle me reçut et même chaque fois que pour la ménager je me retirais elle se rapprochait avec frénésie et saisissant ma pine à pleines mains elle l'enfonçait dans une étreinte encore plus profonde si bien que Hercule! j'aurais même pu croire que pour la faire jouir complètement il me manquait encore quelque chose ah! que la mère du Minotaure et son mugissant amant (BP, p. 622-623, italiques de Simon)

C'est, de toute évidence, le visage d'une femme qui déborde de désir, effrayante et attirante en même temps. C'est à une telle femme que rêve Bernard, en jouant sur le contraste entre celle-ci et la mère frigide qui l'avait conçu entre les bras d'un mari qu'elle n'aimait pas: «Un bâtard, lui, peut se 
dire enfant de l'amour et rêver sur son père, rêver de père inconnu, de cette nuit, de l'étreinte sauvage, clandestine, où il fut conçu, du désir: elle s'ouvrant, recevant, éperdue, fille perdue, fille folle, balbutiant, gémissant...» (Le Sacre du printemps, p. 15).

On pensera aussi à Rose, la serveuse au bon cœur et à la moralité douteuse du Vent, dont la mort sera indirectement causée par Montès. C'est la même femme qui, sous les traits de Sabine, a "tant de fois gémi, forniqué en esprit " (Herbe, p. 141), et celle aussi dont on devine "les imperceptibles sanglots » dans la nuit du Palace (p. 514). Comme l'a si bien dit le philosophe de l'érotisme:

La femme dans les mains de celui qui l'assaille est dépossédée de son être. Elle perd, avec sa pudeur, cette ferme barrière qui, la séparant d'autrui, la rendrait impénétrable: brusquement elle s'ouvre à la violence du jeu sexuel déchaîné dans les organes de la reproduction, elle s'ouvre à la violence impersonnelle qui la déborde du dehors. (Georges Bataille, op. cit., p. 100)

Car c'est bien cette même femme, sexe-gouffre, qui cèdera à l'orage de la violence masculine, aspirée qu'elle sera par la contagion de cette « rage des chiens " dont l'analogie avec les transports amoureux a frappé Bataille, et qui offre aux amants honnêtes "la possibilité d'un dénivellement vertigineux " vers les bassesses vicieuses et ordurières de la pègre où ils pourront constater, après Baudelaire, que " la volupté unique et suprême de l'amour gît dans la certitude de faire le Mal» (ibid., p. 116, 152, 267). C'est à la lumière de ces propos que l'on saisira la portée des mentions discrètes de fellatio (dans La Bataille de Pharsale, p. 593) et de cunnilinctus (voir Orion aveugle, p. 98), et que l'on comprendra mieux la femme-putain, simonienne par excellence, Corinne (de La Route des Flandres), dont il est dit: "vierge, il y avait belle lurette qu'elle ne l'était plus» ( $R F$, p. 200), ce qui explique pourquoi, entre les bras du jockey qui montait les chevaux de son mari, " c'était elle qui écartait les cuisses chevauchait, tous deux chevauchant (ou plutôt qui avaient été chevauchés par) la même houri la même haletante hoquetante haquenée" (p. 399).

Mais au-delà de la femme concrète, particulière, c'est son sexe qui fascine, hante et finalement obsède le narrateur simonien, comme le prouve l'impressionnante variété de synonymes que le lecteur peut trouver pour décrire cet organe troublant entre tous. C'est tantôt une plaie, ouverte au centre du corps de la femme étendue; c'est Louise après son ultime étreinte avec l'amant qu'elle vient de congédier, ne faisant pas un geste pour couvrir 
"l'étroite bouche mauve pâle, semblable à un délicat pétale froissé, une permanente, éternelle et inguérissable blessure» (Herbe, p. 136). Tantôt c'est un "antre [...] broussailleux" (Femmes, p. 15 [La Chevelure de Bérénice, p. 558]), tantôt un « suave et tendre et vertigineux et broussailleux et secret repli de la chair " $(R F$, p. 200), tantôt une moule, " un coquillage au goût de sel » (Hist., p. 226), et tantôt - tout simplement - une bouche, "fendu[e], ovale, humide et entouré[e] de poils » (BP, p. 570). Par ailleurs, nombre de substituts désignent avec insistance l'érotique féminin; substituts animés, comme les pigeons du Palace, " avec leurs jabots gonflés, leurs chatoyantes collerettes de reflets d'un rouge cuivré, irisé » (p. 548) ou inanimés, telles ces feuilles de journal "sitôt tombées s'ouvrant, se détendant d'une brève et courte secousse, comme un dernier sursaut, une dernière respiration " $(P$, p. 549), images désignant avec insistance le coït.

Si le sexe de la femme est récepteur, accueillant ou parfois sinistrement étouffant, comme un poulpe ( $R F$, p. 220), celui de l'homme est naturellement entreprenant et agressif. C'est un «bélier au front cyclopéen et aveugle " (Gul., p. 171), un "boutoir» (Herbe, p. 141), un "fuseau " $(R F$, p. 395), et même un " pilon» (BP, p. 599). Voici avec quel amour complaisant il est décrit dans La Bataille de Pharsale:

Au bout du membre raidi et tendu, la peau du fourreau, d'un léger bistre, découvre à demi le gland, l'entourant d'une couronne au milieu de laquelle saille la pointe, en forme d'ogive, d'une teinte rose et percée au centre de son orifice, comme un œil aveugle. (p. 702)

Mais cet organe, si fier et plein d'arrogance, reste cependant vulnérable et sujet au déclin, comme le montre une allusion significative aux "attributs aujourd'hui pendouillants et ridés de sa défunte virilité " (Hist., p. 176). En attendant le sort qui est réservé à toutes choses par "l'incohérent, nonchalant, impersonnel et destructeur travail du temps " $(R F, \mathrm{p} .412)$, cet élément si puissant dans l'univers simonien, le pénis, est assimilé au monde animal (" un poisson ", ibid., p. 395), végétal (" un vulgaire terreau, enfonçant en lui ses racines ", Herbe, p. 71), et même - à l'instar de l'érotique flaubertienne si finement analysée par Jean-Pierre Richard dans Littérature et sensation - au monde minéral: "puis rien d'autre qu'une unique goutte se gonflant, s'étirant en poire, se distendant, se détachant, allant s'écraser exactement sur la coulée calcaire" (Hist., p. 171).

Ces images - puissamment évocatrices et troubles - des organes de la reproduction, vont de pair avec des tableaux de plus en plus détaillés de l'étreinte amoureuse elle-même. Déjà, dans La Route des Flandres, nous tom- 
bons sur cette description lancinante qui imite si savamment le rythme de l'acte décrit en termes peu voilés:

je roulai sur elle l'écrasant de mon poids mais je tremblais trop fébrile tâtonnant à la recherche de sa chair de l'entrée de l'ouverture de sa chair parmi l'emmêlement cette moiteur légère touffue mon doigt maladroit essayant de les diviser aveugle mais trop pressé trop tremblant alors elle le mit elle-même une de ses mains se glissant entre nos deux ventres écartant les lèvres du majeur et de l'annulaire en $V$ tandis que quittant mon cou son autre bras semblait ramper le long d'elle-même comme un animal comme un col de cygne invertébré se faufilant le long de la hanche de Léda (ou quel autre oiseau symbolique de l'impudique de l'orgueilleuse oui le paon sur le rideau de filet retombé sa queue chamarrée d'yeux se balançant oscillant mystérieux) et à la fin contournant passant sous sa fesse repliée m’atteignant le poignet retourné posant sa paume renversée à plat sur moi comme pour me repousser mais à peine contenant mon impatience, puis le prenant l'introduisant l'enfouissant l'engloutissant respirant très fort elle ramena ses deux bras, le droit entourant mon cou le gauche pressant mes reins où se nouaient ses pieds, respirant de plus en plus vite maintenant le souffle coupé chaque fois que je retombais la heurtais l'écrasais sous mon poids m'éloignant et la heurtant elle rebondissait vers moi et à un moment il sortit mais elle le remit très vite cette fois d'une seule main sans lâcher mon cou, maintenant elle haletait gémissait pas très fort mais d'une façon continue sa voix changée tout autre que je ne connaissais pas c'est-à-dire comme si c'était une autre une inconnue enfantine désarmée gémissant se faisant entendre à travers elle quelque chose d'un peu effrayé plaintif égaré je dis Est-ce que je t’aime? Je la heurtai le cri heurtant sa gorge étranglé elle parvint pourtant à dire:

Non

Je dis de nouveau Tu ne crois pas que je t'aime, la heurtant de nouveau mes reins mon ventre la heurtant la frappant de nouveau tout au fond d'elle sa gorge s'étranglant un moment elle fut incapable de parler mais à la fin elle réussit à dire une seconde fois:

Non

et moi: Tu ne crois pas que je t'aime Vraiment Tu ne crois pas que je t’aime Alors est-ce que je t'aime maintenant Est-ce que je t'aime dis? la heurtant chaque fois plus fort ne lui laissant pas le temps la force de répondre sa gorge son cou ne laissant plus passer qu'un son inarticulé mais sa tête roulant furieusement à droite et à gauche sur l'oreiller parmi la tache sombre de ses cheveux faisant Non Non Non Non, $(R F$, p. 376-377)

J'ai cité ce long passage en entier, non parce qu'il est plus explicite que d'autres (ceux de La Bataille de Pharsale, p. 723-724 et d'Orion aveugle sont presque cliniques dans leur précision) mais parce qu'il me semble d'une beauté plastique indéniable et qu'il nous servira comme exemple et point de repère dans ce qui suit. Pour l'instant il nous suffira de retenir avec quelle poésie le narrateur simonien traite ce sujet délicat entre tous, et avec quelle force il parle de la puissance de l'érotisme, qui sur le plan humain doit être assimilé, comme l'ont successivement démontré Bachelard et Bataille, aux forces à la fois destructrices et fécondantes du monde naturel, telles que la mer, l'orage et le vent. Ce ne peut être pure coïncidence que ce dernier élé- 
ment serve à la fois comme thème et symbole dans le roman de 1957 auquel il a fourni le titre.

En effet, l'érotisme n'est pas restreint, chez Claude Simon, à une insistance sur les organes et leur usage tant normal que perverti (nous employons de toute évidence ces mots dans un sens scientifique, sans leur donner aucune coloration morale ou péjorative). D'autres domaines tombent sous l'influence universelle. Peinture et priapisme, par exemple, sont étroitement associés dans l'œuvre simonienne comme dans la gravure de Picasso. Les ébats des amants de La Bataille de Pharsale sont décrits comme s'il s'agissait d'une création picturale, brossée au pinceau:

Le membre de l'homme, tout entier sorti de la femme maintenant, est représenté d'une façon schématique, arqué vers le haut, plus étroit à sa base qu'à son extrémité, le gland figuré par un triangle à peu près équilatéral pourvu d'un point près de son sommet. (p. 729)

Et dans le même roman, la célèbre bataille romaine est comparée au music-hall et aux « bataillons de girls levant et abaissant leurs jambes - ou leurs bustes - l'un après l'autre de sorte que de longues vagues semblent courir le long du front des cuisses nues, des sourires figés et des coiffures emplumées " (p. 636). Lélément érotique du catch y est également souligné, avec ses " accouplements vaguement obscènes " (p. 653), de même que la sottise affligeante, "équivalente dans l'érotisme à celle des films habituels sentimentaux ou d'aventure » du cinéma de bordel (p. 666). Dans ces trois derniers cas, le narrateur fait ressortir le côté obscène ou l'aspect scabreux d'une activité - la guerre, le catch, le cinéma - considérée habituellement comme appartenant à un univers différent. C'est partout la violence de l'érotisme que l'on met brutalement en lumière, érotisme teinté d'humour noir au spectacle du jaloux à l'affût des bruits d'une étreinte et qui frappe si fort à la porte de la chambre des amants qu'il se casse un os de la main, tandis que Pasiphaé dans sa frénésie se fait presque éventrer par le bovin dont elle jouit (voir BP, p. 623 et p. 677). Car « essentiellement ", affirme si justement Georges Bataille, "le domaine de l'érotisme est le domaine de la violence, le domaine de la violation », sans lesquelles «l'amour sexuel n'aurait pu, comme il l'a fait, prêter son vocabulaire aux descriptions de l'extase des mystiques» (op. cit., p. 21 et p. 265). 
Ce qui nous amène tout naturellement à cette notion-clé chez Bataille de la transgression des interdits, car sans l'élément de la honte du sexe il ne peut y avoir plaisir érotique (horreur de la jouissance et jouissance de l'horreur), dans ce domaine où « le jeu alternatif de l'interdit et de la transgression est le plus clair " (ibid., p. 78). Le sentiment de la culpabilité, de la honte, et la certitude d'avoir transgressé un interdit (BP, p. 653), sont toujours présents à l'esprit du narrateur simonien. Rejeté par Corinne, qui l'accuse de l'avoir traitée comme " une fille à soldats", le narrateur de La Route des Flandres, tout en se sachant innocent de ce reproche, ne peut s'empêcher de se sentir lourdement coupable vis-à-vis de la jeune femme, bien que celle-ci par le passé n'ait point hésité à tromper son mari avec une impudeur révoltante. On trouve dans cette réaction du narrateur les deux notions intimement liées d'éros et de culpabilité. En fait chez tous les personnages de Claude Simon, il y a une conscience aiguë de la parenté profonde entre l'amour et la mort; ce cri de sainte Thérèse, au moment de l'extase, "Je meurs de ne pas mourir! ", qui mieux que Bataille a su mettre en lumière son sens profond?

Nul ne saurait nier qu'un élément essentiel de l'excitation est le sentiment de perdre pied, de chavirer. L'amour n'est pas ou il est en nous, comme la mort, un mouvement de perte rapide, glissant vite à la tragédie, et ne s'arrêtant que dans la mort. Tant il est vrai qu'entre la mort, et la "petite mort ", ou le chavirement, qui enivrent, la distance est insensible. Ce désir de chavirer, qui travaille intimement chaque être humain, diffère néanmoins du désir de mourir en ce qu'il est ambigu: c'est le désir de mourir sans doute, mais c'est en même temps le désir de vivre, aux limites du possible et de l'impossible, avec une intensité toujours plus grande. (Op. cit., p. 262)

Le paroxysme en lequel culmine toute étreinte, et que Bataille qualifie ici de " petite mort ", est assimilé par le narrateur de L'Herbe à " une courte mort, un brusque anéantissement » (p. 78), qui nous renvoie à cette image de l'œil renversé chez Bataille. Il est frappant d'observer à quel point les termes, ainsi que les idées, des deux écrivains sont proches les uns des autres. Les deux événements capitaux de l'expérience humaine - le coït et l'agonie - vont tellement de pair que c'est sans surprise que l'on accepte la façon dont le narrateur d'Histoire évoque le sort des amants engouffrés au moment de l'accouplement par les cendres des cataclysmes de Pompéi et d'Herculanum (p. 216), pas plus que l'on ne s'étonne de la manière dont le jeu sexuel se trouve assimilé au combat fratricide livré autrefois sur une colline pierreuse de Thessalie:

maintenant il s'élance et presqu'aussitôt il reçoit dans la bouche un coup de glaive dont la pointe ressort par la nuque non pas la mort mais le sentiment de ta mort je ne savais pas encore 
se courbant sa broussaille de poils jaunes frottant le bout de ses seins elle arquée son corps reposant seulement sur ses épaules et la plante abricot de ses pieds à plat sur le lit les reins soulevés les jambes dans la position de ces acrobates faisant le pont

à présent le court morceau de flèche ou de javeline brisée qui sort de son dos projette une ombre qui s'étire traverse en diagonale l'omoplate s'allonge encore rejoint la zone obscure où disparaissent sa tête et ses bras la lumière rasante indiquant qu'il est tard dans l'après-midi se traînant n'en finissant plus le soleil

la cavalerie est repoussée les archers et les frondeurs sont taillés en pièces

maintenant elle entoure ses épaules de ses bras

dans la confusion des voix crièrent À cheval Avec un bruit d'air froissé le pigeon passa devant le soleil ailes déployées Jaune puis forme d'arbalète noire puis jaune de nouveau aveuglant sable sous les paupières

un de mes bras passé sous ses épaules l'autre la tenant par-dessous elle ruisselait entre ses fesses mon doigt glissait dans (BP, p. 641)

La lutte des corps, en effet, qu'elle soit amoureuse ou mortelle, est une métaphore primordiale dans l'œuvre de Claude Simon. Le narrateur de La Route des Flandres cherche à travers son étreinte avec Corinne, dont j'ai cité plus haut la description, à percer le mystère des de Reixach, de l'aïeul tué par une balle dans on ne sait quelles circonstances historiques, et du descendant fauché par une mitrailleuse allemande à la bataille de la Meuse; mais cette recherche est finalement vaine, car si, comme disait Ronsard, "l'amour et la mort n'est qu'une mesme chose ", l'une n'expliquera jamais l'autre et n'en éclaircira jamais les ténèbres.

Dans cette perspective, à propos de cette œuvre où la naissance frôle la mort et inversement (Le Palace, p. 549-550), et où plane l'odeur de cadavre (Histoire, passim), peut-on parler de cette "approbation de la vie jusque dans la mort " qui est pour Georges Bataille, le summum de l'érotisme? Approbation qui, en dépit de toutes les nuances restrictives qu'il convient d'y apporter, gît à la base des romans de D.-H. Lawrence? "Voilà ce que leur amour était devenu ", peut-on lire dans L'Arc-en-ciel, " une sensualité violente et extrême comme la mort. Il n'y avait entre eux aucune conscience d'intimité, aucune tendresse amoureuse. Tout était luxure, intoxication délirante des sens, passion de mort ». Les amants de Lawrence baignent dans l'extase exacerbée de la "Beauté Absolue ", où le plaisir est si extrême qu'il se transmue presque en douleur. Mais cette vue de l'esprit reste profondément optimiste, car chez Lawrence, on peut, normalement, se découvrir et se réaliser à travers l'expérience érotique poursuivie avec tant d'entrain et d'acharnement qu'elle ne se distingue plus d'un mysticisme quasi religieux. Les personnages du grand romancier anglais souffrent, bien entendu, de ce que Georges Bataille appellerait leur « discontinuité », car ils sont conscients 
d'être des individus " mourant isolément dans une aventure inintelligible ", ayant la nostalgie de la continuité perdue (op. cit., p. 20). Mais à la différence des personnages simoniens ils peuvent sous certaines conditions, se dépasser et s'accomplir dans l'érotisme. Si j'ai bien lu les romans de Claude Simon, cette forme d'approbation ne s'y discerne point. Je ne puis oublier cette image, d'une terrifiante beauté, où le spasme amoureux est directement assimilé à l'acte homicide, la femme qui jouit sous les caresses de son amant et le légionnaire qui meurt sous les coups de son adversaire ne faisant qu'un:

pilum frappant entrant et ressortant à plusieurs reprises de la blessure le renflement de sa pointe triangulaire arrachant aux lèvres le sang jaillissant par saccades brûlant Elle m'inonda se mit à hoqueter et crier balbutiant des mots sans suite donnant de violents coups de reins (BP, p. 589)

Car l'érotisme simonien - à l'inverse de l'érotisme de Lawrence, de Durrel, de Robbe-Grillet même - n'est guère optimiste. C'est un érotisme désespéré, intimement lié, comme chez le marquis de Sade et ses épigones romantiques, à l'idée de la mort. L'univers viscéral, foisonnant, organique de Claude Simon est hanté, en définitive, par cette constatation tragique, que l'homme, emprisonné dans la durée, sujet aux incohérents délabrements du temps, à cet « illusoire et apaisant afflux » dont parle Le Tricheur (p. 87), tente désespérément, mais finalement en vain, de s'en délivrer par le truchement du dépassement érotique. Mais il lui reste une lueur d'espoir, la création artistique: peinture et priapisme, érotisme et écriture, nous l'avons vu, ne font qu'un dans cette œuvre baroque et splendide. À l'instar de Samuel Beckett, peut-être Claude Simon a-t-il réussi à arracher du néant auquel est voué tout élan humain, l'unique consolation qui nous demeure possible: la contemplation de l'œuvre d'art qui consacre notre irrémédiable et inéluctable faillite. 